University of Nebraska - Lincoln

DigitalCommons@University of Nebraska - Lincoln

Faculty Publications in Computer \& Electronics Electrical \& Computer Engineering, Department Engineering (to 2015)

2008

\title{
Combined User Multiplexing and Data Modulation through Non- binary Turbo Codes for UWB
}

\author{
K. Popovski \\ University of Wollongong \\ Tadeusz Wysocki \\ University of Nebraska-Lincoln, wysocki@uow.edu.au \\ B. J. Wysocki \\ University of Nebraska-Lincoln
}

Follow this and additional works at: https://digitalcommons.unl.edu/computerelectronicfacpub

Part of the Computer Engineering Commons

\footnotetext{
Popovski, K.; Wysocki, Tadeusz; and Wysocki, B. J., "Combined User Multiplexing and Data Modulation through Non-binary Turbo Codes for UWB" (2008). Faculty Publications in Computer \& Electronics Engineering (to 2015). 37.

https://digitalcommons.unl.edu/computerelectronicfacpub/37
}

This Article is brought to you for free and open access by the Electrical \& Computer Engineering, Department of at DigitalCommons@University of Nebraska - Lincoln. It has been accepted for inclusion in Faculty Publications in Computer \& Electronics Engineering (to 2015) by an authorized administrator of DigitalCommons@University of Nebraska - Lincoln. 


\title{
Combined User Multiplexing and Data Modulation through Non-binary Turbo Codes for UWB
}

\author{
K. Popovski ${ }^{\dagger}$, Member, IEEE, T. A. Wysocki ${ }^{\dagger \dagger}$, Senior Member, IEEE, and B. J. Wysocki ${ }^{\dagger}$ \\ ${ }^{\dagger}$ School of Electrical, Computer and Telecommunications Engineering \\ University of Wollongong, Northfields Ave, Wollongong, NSW, 2522, Australia \\ Email: \{keni, bjw\}@uow.edu.au \\ ${ }^{\dagger \dagger}$ Department of Computer and Electronics Engineering \\ University of Nebraska - Lincoln, 1110 South 67 St, Omaha, NE, 68182-0572, United States of America \\ Email: twysocki@mail.unomaha.edu
}

\begin{abstract}
With significant research being conducted in Ultra Wideband communications to increase error performance, this paper proposes a combination of user multiplexing and data encoding to achieve a BER improvement. Through the use of non-binary turbo coding, data is modulated to form a time hopping code which is applied to a time-reversed UWB system. Comparative results with conventional binary coding are given, showing the possibility for BER improvements in systems with a low number of users. Slight performance degradations for a large number of users exist. The effects of a user applying turbo coding on other users not applying forward error correction is also studied.
\end{abstract}

Index Terms-UWB, time-reversed, pre-rake equalization, TCM, TTCM, Nonbinary-Turbo

\section{INTRODUCTION}

$\mathbf{U}$ LTRA Wideband (UWB) has seen increased attention since its release for commercial applications in early 2002 [1]. It is characterized by having a fractional bandwidth of more than $20 \%$, or bandwidth occupancy greater than 500 $\mathrm{MHz}$ [2]. Its integration into mainstream wireless communications is evident with Intel's adoption of UWB for high-speed interconnections of home and office digital equipment with its Wireless UWB Link 1480 Media Access Controller [3].

The two main competitors for the UWB standard are direct sequence based and orthogonal frequency division multiplexing based schemes, supported by the 'UWB Forum' and 'WiMedia Alliance' respectively. This paper is focused on time reversed UWB (TR-UWB) [4], similar in implementation to direct sequence UWB. This scheme has also been referred to as 'pre-rake' [5]. While a conventional system would operate with the transmission of sub-nanosecond width Gaussian waveforms, a TR-UWB system uses the channel impulse response from the transmitter to the receiver as a transmit pre-filter.

Traditionally, pulses transmitted are either delayed in time (pulse position modulation (PPM)) or changed in amplitude (pulse amplitude modulation (PAM)) for encoding data, and users are multiplexed through code division multiple access based upon a family of orthogonal time hopping codes. Turbo coding may also be applied in order to attain a performance closer to the capacity of the communication channel. This paper explores the combination of data encoding with time hopping, achieved through the use of non-binary turbo codes. It is proposed to encode information symbols onto an expanded modulation set, rather than binary pulse encoding combined with preset time hopping codes. Trellis turbo coded modulation (TTCM) is utilized, which was first presented by Robertson and Worz [6].

Similarly to binary turbo codes, TTCM uses a parallel concatenation of two binary TCM encoders. TCM combines rate $R=m /(m+1)$ binary convolutional codes with an $M$-ary signal constellation, optimizing the Euclidean distance between codewords [7]. By replacing the component codes in a binary encoder with trellis codes, coding gains are achievable without a subsequent bandwidth expansion [8]. Increased performance is possible relative to classical binary coding, with TTCM having a better convergence of the iterative decoding, less sensitivity to puncturing patterns, and greater robustness toward the flaws of the decoding algorithm [9].

A memoryless mapper generates a one-to-one correspondence between the coded bits and the time hopping chip positions. Decoding is conducted symbol by symbol, rather than bit by bit as in conventional turbo coding. A modified symbol based SOVA decoding algorithm is applied, based upon maximum likelihood accumulated metrics through the trellis. An additional degree of user differentiation is achieved through a random PPM shifting in the TTCM system.

This paper is organized as follows: Section II presents an overview of the UWB system architecture studied, together with the system equations for conventional binary turbo coding. Section III considers the application of TTCM for time hopping based data encoding, with comparative results shown in Section IV. Concluding statements are given in Section V.

\section{UWB ARCHITECTURE}

\section{A. TR-UWB System Equations}

The complete TR-UWB system is presented in [10], with an overview of the system equations presented here. The signal $x^{(u)}(t)$ transmitted for the $u$ th user in a time-hopped timereversed UWB system, with the time shift $\varepsilon$ set to equal the 
pulse width, and equiprobable data $b_{j}^{(u)} \in\{-1,1\}$ mapped through binary PPM, is given by:

$$
\begin{array}{r}
x^{(u)}(t)=\frac{\sqrt{E_{T X}(u)}}{\sqrt{G_{H, u ; x_{\text {pos }}}}}\left(\sum_{j=0}^{n-1} w\left(t-j T_{f}-c_{j}^{(u)} T_{c}-\varepsilon b_{j}^{(u)}\right)\right) \\
\otimes h\left(u ; x_{p o s},-t\right),
\end{array}
$$

where $\otimes$ represents convolution, $E_{T X}(u)$ is the user signal energy, $G_{H, u ; x_{p o s}}$ represents the gain of the channel required for normalization, $w(t)$ is the base transmitted waveform of width $T_{w}$ seconds, $j$ is the frame number, and $x_{\text {pos }}$ represents the position of the receiver. $T_{f}$ is a single frame length, which is segmented into $N_{h}$ equally spaced intervals called 'chips' of duration $T_{c}$, such that $T_{f}=N_{h} T_{c} . c_{j}^{(u)}$ denotes the position within the particular frame (the chip number) that is occupied by the $u$ th user's signal in accordance with a time hopping sequence. It should be noted that a perfectly power controlled system is assumed, whereby $E_{T X}$ is constant for all users. The transmitted signal is pre-filtered using the time-reversed complex conjugate of the forward link channel response.

The signal received is defined as:

$$
y(t)=\left(\sum_{u=1}^{N_{u}} x^{(u)}(t) \otimes h\left(u ; x_{\text {pos }}, t\right)\right)+n(t),
$$

where a summation takes to account contributions of all $N_{u}$ users. All transmitters were assumed dispersed enough such that the channel responses from each $N_{u}$ transmitter to any receiver are independent. As such, each convolution is calculated using the response from user $u$ to the desired receiver. Additive white Gaussian noise with variance of $\sigma^{2}=N_{0} / 2$ is also present.

The base pulse $w(t)$ was set as the second derivative of the Gaussian pulse, with center frequency $f_{0}$, defined as [11]:

$$
w(t)=\left[1-2\left(\pi t f_{0}\right)^{2}\right] \exp \left\{-\left(\pi t f_{0}\right)^{2}\right\} .
$$

A center frequency of $3.9 \mathrm{GHz}$ was used, which results in a monocycle width of $T_{w}=0.5 \mathrm{~ns}$.

For experimentation within this paper, a chip synchronous single-input-single-output system is considered, assuming transmit and receive antennas, which would act as pulse shaping filters, have no significant effect on the signal transmitted. Time reversal properties still apply in a SISO system, assuming that the bandwidth occupied by transmissions is much larger than the correlation frequency exhibited by the channel [12].

The multipath model applied is the IEEE 802.15.3a channel, based on the SV model where multipath components arrive in clusters [13]. The LOS channel scenario (CM1) was selected for all testing within this paper. A quasi-stationary channel is assumed, remaining time-invariant for the transmission of a block of data, and independent between blocks.

\section{B. SOVA-based Turbo Coding}

The addition of turbo coding into a UWB system operates at the binary data level, altering $b_{j}^{(u)}$ in accordance with a preset convolutional code. This code is based on passing the data through a set of linear shift registers, such that $n$ encoder outputs depend on both $k$ encoder inputs and $\gamma$ previous inputs [14]. The structure of the convolutional encoder is defined by a generator matrix in the form $G(D)=\left[g_{1}, g_{2}\right]$, with $g_{1}$ and $g_{2}$ representing the feedforward and feedback components of the recursive systematic convolutional (RSC) encoder respectively.

The turbo transmitter consists of two RSC encoders, generally with the same generator matrix, operating on the same binary input data. However, the second encoder block operates on the permuted data representation [15]. The combined result is a systematic output sequence, together with two parity outputs which have same length as the input. This gives an overall code rate $R=k / n=1 / 3$.

Higher data rates may be achieved by puncturing the filter outputs before multiplexing, alternately selecting between the two possible parity values. This forms a $P=\left[\begin{array}{lll}1 & 0 ; 0 & 1\end{array}\right]$ puncturing scenario, where the column indicates the RSC encoder activated at each time instant. This results in a code rate of $R=1 / 2$.

Termination bits are appended to the transmitted data sequence in order to force the first RSC component encoder to end in a known state. The addition of these $\gamma$ tail bits does not control the final state of the second component encoder however, as it operates on the permuted data sequence. Methods such as circular termination of the component codes or 'tailbiting' exist such that additional termination bits are not required [16].

The size of the interleaver is determined by the parameter $n$, with larger interleaver sizes generally associated with lower error rates. The interleaver spreads adjacent symbols in the transmission, making them independent of adjacent symbols in reception [14]. This reduces the effects of burst errors and impulse noise.

Through correlation of the PPM signal with a static template, the receiver front-end produces a magnitude representation for each bit in the encoded data stream. This measure indicates the likelihood that the bit is a 0 or a 1 . A negative value indicates a bit ' 0 ', a positive value indicates a bit ' 1 ', and a value near zero shows equal possibility of either data bit.

The turbo receiver structure consists of separate decoding for each of the constituent codes, together with the iterative exchange of reliability information [14]. Each soft-input-softoutput (SISO) decoder produces extrinsic probabilities on the transmitted data, which is permuted to give the a priori information. This is passed to the next decoder which operates on the next parity sub-block, together with the same systematic data. Each iteration seeks to improve the log-likelihood ratio (LLR) $L\left(d_{k}\right)$, where $d_{k}$ is the $k$ th received data bit, converging to an estimate of the transmitted codeword. For a binary symmetric channel, the LLR is defined as [17]:

$$
L\left(d_{k}\right) \triangleq \log \frac{\operatorname{Pr}\left(d_{k}=+1\right)}{\operatorname{Pr}\left(d_{k}=-1\right)} .
$$

Representing the sampled received data for an arbitrary user 
as $y_{k}$, the LLR can be altered to:

$$
L\left(d_{k} \mid y\right)=\frac{E_{T X}}{2 \sigma^{2}} 4 a \cdot y_{k}
$$

where $a$ is the fading amplitude, which has a value of 1 for the Gaussian channel. A 'channel reliability' parameter may be extracted as: $L_{c}=4 a E_{T X} / 2 \sigma^{2}$.

Each decoder initiates the soft-output Viterbi algorithm (SOVA), which extends on the Viterbi algorithm. It uses a modified path metric to take into account the a priori probabilities of the input symbols, and produces a reliability indicator to the hard decision output for each bit [7]. It utilizes Euclidean distance to determine the highest reliability path through the trellis. The branch metric is defined as [15]:

$$
\mu_{t}\left(y_{k}, p, q\right)=\log \operatorname{Pr}\left(y_{k} \mid B^{(p, q)}\right),
$$

where $B^{(p, q)}$ represents the transition in the trellis from arbitrary states $p$ to $q$. The path metric can hence be iteratively calculated as:

$$
M_{t}(q)=M_{t-1}(p)+\mu_{t}\left(y_{k}, p, q\right) .
$$

The incorporation of prior information transforms the path metric to:

$$
M\left(\vec{S}_{k}^{s}\right)=M\left(\vec{S}_{k-1}^{s^{\prime}}\right)+\frac{1}{2} d_{k} L\left(d_{k}\right)+\frac{L_{c}}{2} \sum_{l=1}^{n} y_{k}^{l} x_{k}^{l},
$$

where $y_{k}^{l}$ and $x_{k}^{l}$ represent the $l$ th bit in the $n$ bits of the received and transmitted signals respectively.

Taking $M\left(\vec{S}_{k}^{s}\right)$ and $M\left(\vec{S}_{k}^{s^{\prime}}\right)$ as the metric values of the survivor and the discarded paths terminated at states $S_{k}=s$ and $S_{k}=s^{\prime}$ respectively, the metric difference may be defined as:

$$
\Delta_{k}^{s}=M\left(\vec{S}_{k}^{s}\right)-M\left(\vec{S}_{k}^{s^{\prime}}\right) \geq 0
$$

This metric difference is subsequently equivalent to the LLR for the correct decision path, although it requires the consideration of the effects of all discarded paths. Generally, a delay time of $\delta=5 \gamma$ is taken, where $\gamma$ is the number of shift registers in the RSC encoder. The minimal metric difference over all discarded paths which produce an incorrect bit are calculated, giving the final soft output for the bit $d_{k}$ as [17]:

$$
L\left(d_{k} \mid y\right) \approx d_{k} \min _{\substack{i=k \ldots k+\delta \\ d_{k} \neq d_{k}^{i}}} \Delta_{i}^{s_{i}} .
$$

Thus, the polarity of the LLR is determined by the decoded data bit, while the magnitude is equivalent to the reliability of the decision. The decoder's input is subtracted from this soft output in order to prevent the decoder acting as a positive feedback amplifier [16], giving:

$$
L_{e}\left(d_{k}\right)=L\left(d_{k} \mid y\right)-L\left(d_{k}\right)-L_{c} y_{k}^{s} .
$$

This extrinsic information is then interleaved/deinterleaved to give the a priori information for the alternate decoder.

It should be noted that with a punctured code, ignoring tailing bits, double the number of pulses will be transmitted relative to a TR-UWB system operating at the same data rate. For comparability, the energy level of each pulse is halved.

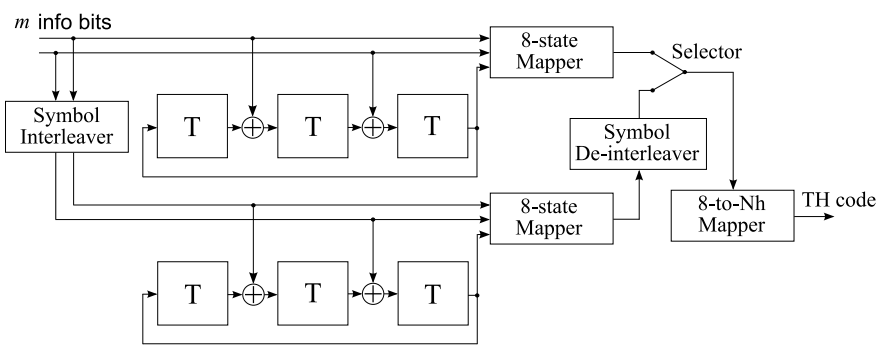

Fig. 1. TTCM transmitter structure

\section{TTCM SYSTEM INTEGRATION}

\section{A. TTCM Transmitter}

The incorporation of a TTCM transmitter into a UWB system allows the combination of the time hopping stage with the data encoding state. This entails the determination of $c_{j}^{(u)}$ using, in this paper, $m=2$ data bits. This generates an 8 constellation point output. The structure of a TTCM transmitter is illustrated in Fig. 1. Similarity to a conventional turbo transmitter is evident, although the parallel concatenation of two identical $m$-ary RSC encoders here operates on $m$-bitwords. The $2^{m}$ possible inputs are encoded using a code rate of $m / m+1$, with the encoded $m+1$ bits mapped to one of $2^{(m+1)}$ constellation points [8], [18]. This $m+1$ bit output, with $m=2$, consists of two systematic bits, together with a single parity bit. The encoder selects the symbols alternately from the two TCM encoders, resulting in an aligned systematic component between the encoders, with only the parity bit being alternately chosen. A total of $\gamma=m+1$ shift registers are utilized in each RSC encoder.

Constraints imposed on the component code include that no parallel transitions should exist in the corresponding trellis diagram of the convolutional encoder. This is to ensure each data bit benefits from the interleaving and parallel concatenation. Also, the information bits in an arbitrary step $k$ should not directly influence the parity bit at step $k$ [6]. The final restriction on the TTCM transmitter is that the interleaver must operate on a pairwise basis, where even blocks of $m$ bits are mapped to even positions, and odd blocks of $m$ bits mapped to odd positions [6], [8], [18]. This symbol interleaver is applied on the data input before processing with the lower RSC encoder, with a de-interleaving operation conducted on the result to ensure the ordering of the systematic component between both encoders.

It can be noted in this TTCM transmitter that trellis termination is not applied, which will inevitably lead to a slight performance degradation relative to a binary turbo system. Also, unlike in conventional turbo, this transmitter always applies symbol puncturing. A histogram based analysis of this punctured output, considering a random interleaver design with a Bernoulli mix input binary stream, proves a uniform probability of the occurrence of each constellation point.

The final block in this structure $\left(8-\right.$ to $-N_{h}$ Mapper) maps the 8 possible constellation points uniquely onto the $N_{h}$ possible chip positions utilized for UWB time hopping. As simulations conducted in this paper are chip synchronous, a random PPM shift $(\varepsilon)$ must be applied in order to diversify 


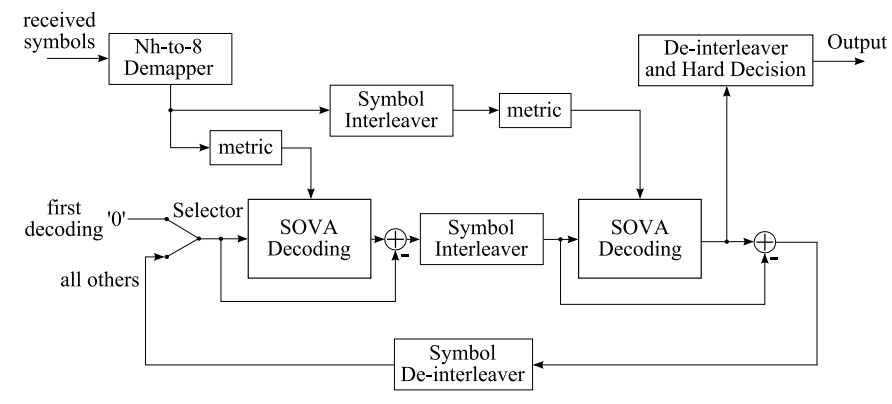

Fig. 2. TTCM receiver structure

the TTCM signals further. This scattering is done based upon the aforementioned 8 point mapper.

Since $m=2$ bits are transmitted per $N_{s}$ pulses (all $N_{s}$ pulses having the same chip position), the number of pulses required for data transmission is effectively halved. For comparable results, the energy of each pulse in this TTCM system is doubled.

\section{B. TTCM Receiver}

1) Decoder Structure: The TTCM receiver is similar in structure to that for binary turbo codes, both based upon an iterative process. However, sets of log-likelihood ratios are passed between the component decoders, rather than single log-likelihoods [19]. The underlying SOVA algorithm is subsequently more complex, as the systematic and extrinsic components cannot be separated since noise simultaneously affects the parity and systematic bits [6]. Here the output is split into two components: the a priori information $\left(L_{a}\right)$ and the combined extrinsic and systematic information $\left(L_{e \& s}\right)$, with the latter being interleaved/de-interleaved and passed between alternate decoders. A 'hard' decision is conducted on the final LLRs to evaluate the symbol that was received.

It can be noted that the performance difference between MAP and SOVA decoding is reduced when non-binary coding is applied [9]. The receiver structure and symbol-based SOVA algorithm with maximum sequence posteriori probability estimations discussed herein is adapted from [18] and [20]. The structure used is shown in Fig. 2.

Transmitting through TR-UWB, the soft data that is passed to the receiver is the correlation of the received signal in each of the $N_{h}$ chips with a preset template of the expected received signal. Collection of correlation values is conducted over all $N_{s}$ transmitted signals. The $2^{m}$ possible transmission locations are then de-mapped. $2^{m}$ a priori values are given a zero neutral probability on the first iteration, since the transmitter utilized puncturing between the component encoders.

2) Symbol Based SOVA: With $\gamma$ shift registers utilized in the encoder, the state of each component encoder can be represented as $S_{k} \in\left\{0,1, \ldots, 2^{\gamma}-1\right\}$. The $m$ bit input is now denoted as $d_{k} \in\left\{0,1, \ldots, 2^{m}-1\right\}$, with received sequence $y_{1 \rightarrow N}=\left(y_{1}, y_{2}, \ldots, y_{N}\right)$ shown from time 1 to $N$. The LLR value for each possible symbol $i$ at time $k$ is calculated as:

$$
L\left(d_{k}=i\right) \triangleq \log \frac{\operatorname{Pr}\left(d_{k}=i \mid y_{1 \rightarrow N}\right)}{\operatorname{Pr}\left(d_{k}=0 \mid y_{1 \rightarrow N}\right)},
$$

with the a priori information being:

$$
L_{a}\left(d_{k}=i\right)=\log \frac{\operatorname{Pr}\left(d_{k}=i\right)}{\operatorname{Pr}\left(d_{k}=0\right)} .
$$

The cumulated path likelihood metric is represented as $M\left(\vec{S}_{k}^{s}\right)=\log \left(\operatorname{Pr}\left(\vec{S}_{k}^{s}, \vec{y}_{t \leq k}\right)\right)$, looking at time $k$ and current state $s$. The state vector is $\vec{S}_{k}^{s}$, which identifies the states along the survivor path terminated at state $S_{k}=s$.

The TTCM receiver trellis thus assumes that $2^{m}$ branches with distinct symbols enter each of the $2^{\gamma}$ trellis nodes. The parameter $q\left(d_{k}=i \mid S_{k-1}=s^{\prime}, S_{k}=s\right)$ is defined as the probability that symbol $i$ is associated with the transition from states $S_{k-1}=s^{\prime}$ to $S_{k}=s$. Beginning with the metric base equation for binary SOVA shown in Eq. 7, and applying the simplification procedure presented in [18], the cumulated path metric may be approximated as:

$$
\begin{aligned}
& M\left(\vec{S}_{k}^{s}\right)=M\left(\vec{S}_{k-1}^{s^{\prime}}\right)+ \\
& \quad L_{c} \cdot \log \left(p\left(y_{k} \mid d_{k}=i, S_{k}=s, S_{k-1}=s^{\prime}\right)\right) \\
& \quad+\left[L_{a}\left(d_{k}=i\right)-\max _{i \in\left(0,1, \ldots, 2^{m}-1\right)}\left(L_{a}\left(d_{k}=i\right)\right)\right],
\end{aligned}
$$

for $q\left(d_{k}=i \mid S_{k-1}=s^{\prime}, S_{k}=s\right)=1$, and:

$$
M\left(\vec{S}_{k}^{s}\right)=-\infty
$$

for $q\left(d_{k}=i \mid S_{k-1}=s^{\prime}, S_{k}=s\right)=0$. The term $\log \left(p\left(y_{k} \mid\right.\right.$ $\left.\left.d_{k}=i, S_{k}=s, S_{k-1}=s^{\prime}\right)\right)$ is calculated from the soft received values. The multiplication by the channel metric $L_{c}$ ensures that the reliance on received soft values is determined by the channel reliability relative to prior reliabilities.

The log likelihood ratio for each symbol is calculated through the maximum likelihood metric obtained through the Viterbi decoding on the $2^{m}$ paths entering each state. The parameter $\Gamma_{k, i}\left(\vec{S}_{N}^{s}\right)$ is defined as the reliability difference between the maximum likelihood symbol at time $k$ and the most likely codeword with $d_{k}=i$, both terminated at state $S_{N}^{s}$ :

$$
\Gamma_{k, i}\left(\vec{S}_{N}^{s}\right)=\max _{\text {all } \vec{S}_{N}^{s}}\left\{M\left(\vec{S}_{N}^{s}\right)\right\}-\max _{\vec{S}_{N}^{s}: d_{k}=i}\left\{M\left(\vec{S}_{N}^{s}\right)\right\} .
$$

The LLR values can be obtained as:

$$
L\left(d_{k}=i\right)=\Gamma_{k, 0}\left(\vec{S}_{N}^{s}\right)-\Gamma_{k, i}\left(\vec{S}_{N}^{s}\right) .
$$

As in the binary SOVA algorithm, to simplify calculations and limit the memory requirements at the receiver, $L\left(d_{k}=i\right)$ is estimated over $\delta=5 \gamma$ trellis increments. Each state in the trellis stores a $\delta \times 2^{m}$ reliability measure matrix, under the assumption that the survivor and competition paths have converged over the $\delta$ time increments. The reliability difference between survivor and competing paths with $d_{k}=l$, and both terminated at state $S_{t+1}^{s}$, is denoted as:

$$
\Delta_{t+1, l}^{s}=\max _{\text {all } \vec{S}_{t+1}^{s}}\left(\vec{S}_{t+1}^{s}\right)-M\left(S_{t, l}, S_{t+1}^{s}\right),
$$

where $M\left(S_{t, l}, S_{t+1}^{s}\right)$ represents the cumulative path metric terminated at state $S_{t+1}^{s}$ and previous state $S_{t, l}$. It can be 


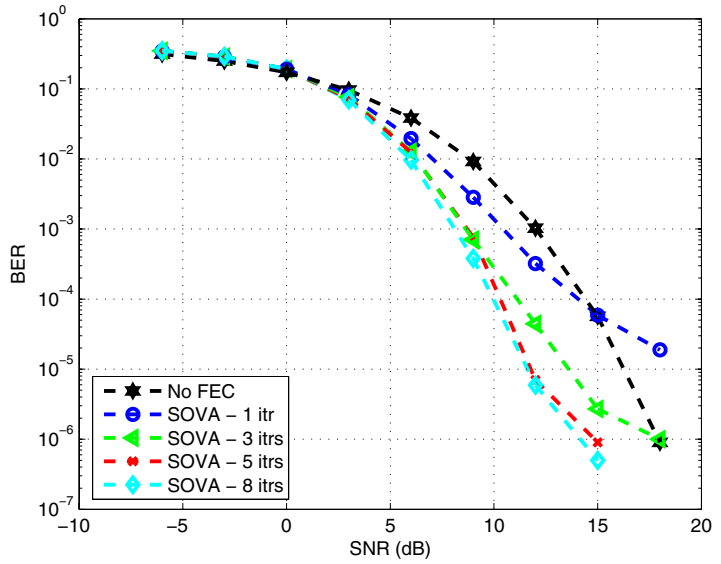

Fig. 3. Binary SOVA scenario, 1 user

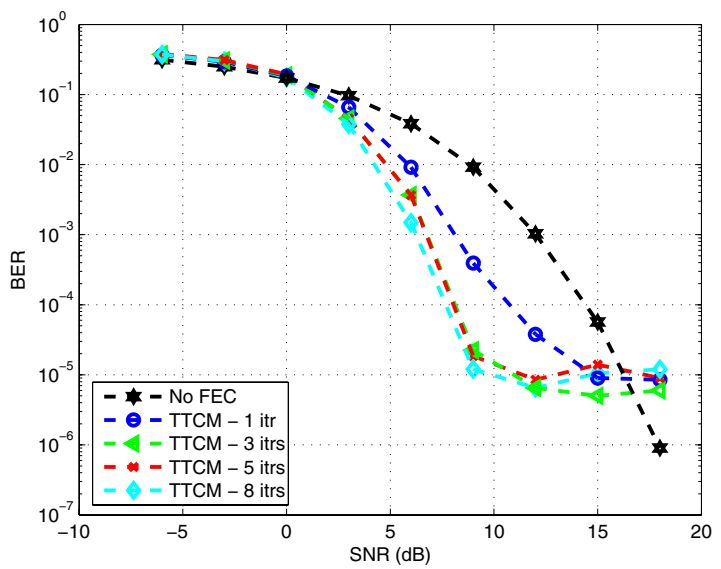

Fig. 4. TTCM scenario, 1 user

noted that $\Delta_{t+1, l}^{s}=0$ for the surviving path at state $S_{t+1}^{s}$. Initially, $\Gamma_{k, i}\left(\vec{S}_{k}^{s}\right)=\Delta_{k, i}^{s}$, with the remaining reliability values $\Gamma_{k, i}\left(\vec{S}_{t+1}^{s}\right)$ for $k=t-\delta+2, \ldots, k$ updated as [18], [20]:

$$
\Gamma_{k, i}\left(\vec{S}_{t+1}^{s}\right)=\min _{l=0,1, \ldots, 2^{m}-1}\left\{\Delta_{t+1, l}^{s}+\Gamma_{k, i}\left(\vec{S}_{t, l}\right)\right\} .
$$

Traversal through the trellis to determine the transmitted signals is similar to the conventional binary turbo method. The LLR value for each $d_{k}=i$ relative to $d_{k}=0$ is subsequently estimated over $\delta$ time positions in the trellis. $L\left(d_{k}=0\right)$ by definition will always give a neutral probability.

\section{Simulation Results}

A TR-UWB simulation was adapted from a time hopped PPM UWB simulation by Di Benedetto and Giancola [21], with a binary SOVA decoder being adapted from [22]. The cardinality and periodicity of each time hopping code were set to 11 , with a pulse width of $0.5 \mathrm{~ns}$, and a data encoding shift of $0.5 n s$. Transmit power was set to $1 \mathrm{~mW}$, with the results scalable. Random interleavers were chosen for both binary and non-binary turbo systems, with the binary system

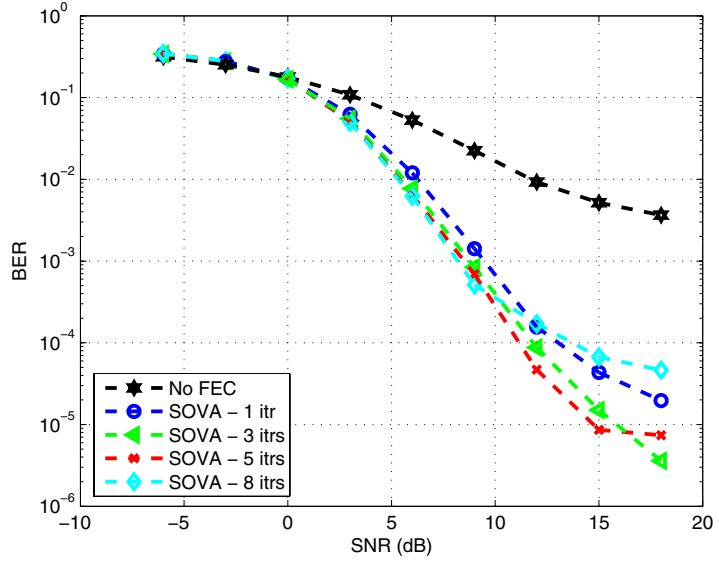

Fig. 5. Binary SOVA scenario, 10 users

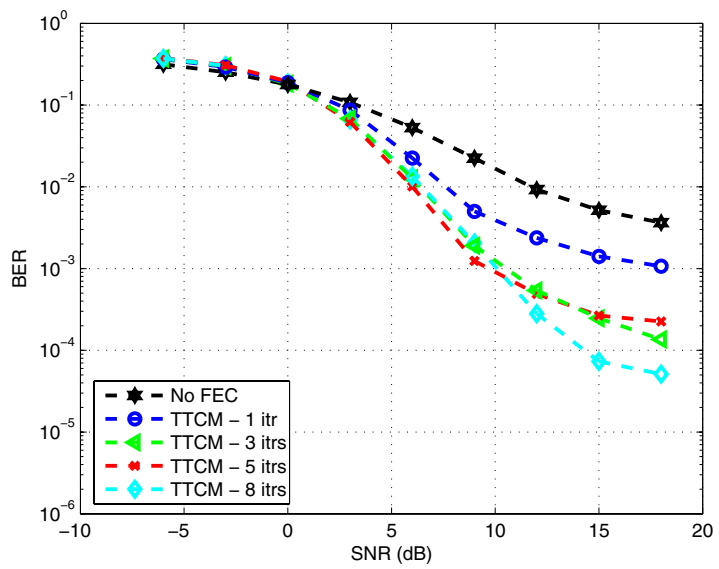

Fig. 6. TTCM scenario, 10 users

applying a puncturing pattern of $P=\left[\begin{array}{llll}1 & 0 ; 0 & 1\end{array}\right]$. The binary SOVA algorithm had a generator matrix with $g_{1}=(101)_{2}$ and $g_{2}=(111)_{2}$. Reed-Solomon time hopping was applied in all cases except in TTCM simulations, with $N_{s}=1$ constant for all tests. A block length of 400 pulses was utilized.

The performances of a single user scenario utilizing binary SOVA and TTCM systems with a data rate of $50 \mathrm{Mbit} / \mathrm{s}$ are depicted in Fig. 3 and Fig. 4 respectively. The error floor for both scenarios is evident, although more prominent in TTCM. It can be seen that in a single user system, TTCM provides a performance advantage over conventional binary SOVA decoding, although requiring a large block size in order to overcome the high SNR error floor.

Shown in Fig. 5 and Fig. 6 are the bit error rates for binary SOVA and TTCM systems operating at 20Mbit/s with 10 users applying error correction. It can be seen that multiple user access interference has a greater impact on TTCM than binary SOVA, although still giving a coding gain of over a decade for a high number of iterations.

In order to observe the effects of TTCM on users not applying error correction, a 10 user system where only one user applied turbo coding was studied. Results for a binary SOVA and TTCM scenario with data rate of $20 \mathrm{Mbit} / \mathrm{s}$ are 


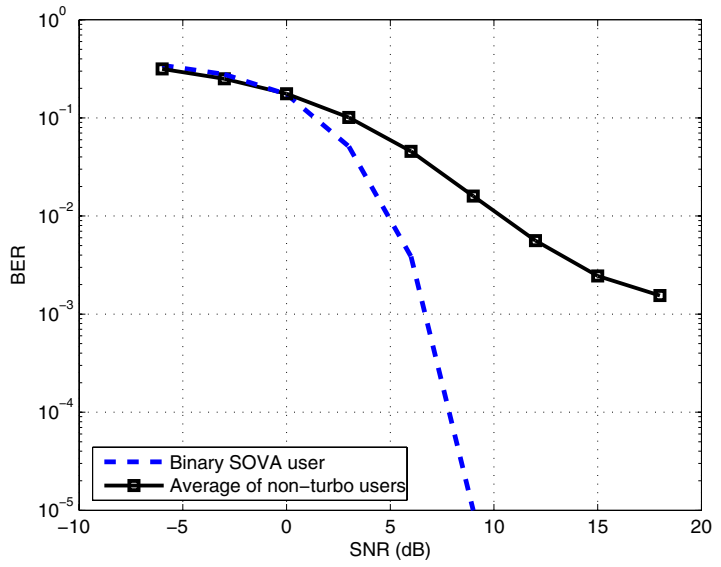

Fig. 7. Binary SOVA scenario, 10 users with single user applying FEC

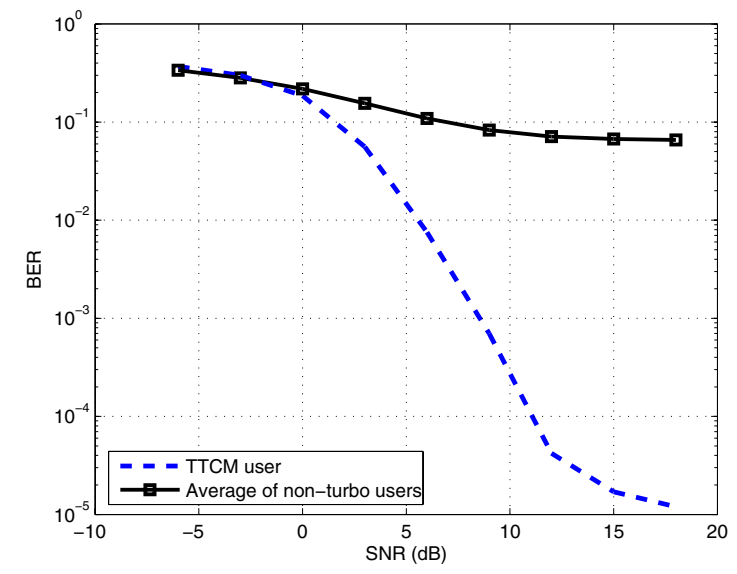

Fig. 8. TTCM scenario, 10 users with single user applying FEC

illustrated in Fig. 7 and Fig. 8 respectively. Turbo coding was applied over 5 iterations. Although transmitting the same symbol energy, the reduced number of pulses for TTCM resulted in a larger energy per pulse. This is the cause of the degradation to non-turbo users relative to the effects of binary SOVA. Also, slightly worse performance was noticed for a user applying TTCM coding rather than conventional binary coding.

\section{CONCLUSions AND Future Work}

The combining of user time hopping with data modulation is proposed, applying non-binary turbo codes with a symbol based decoding algorithm. It was seen that a performance improvement is possible relative to a UWB system not adopting forward error correction. In contrast to conventional binary turbo coding with a SOVA decoder, a bit error rate improvement was noticed for a low number of users, although slight performance degradations for a higher number of interfering users. The increase in single pulse energy resulted in a detrimental effect to users not applying error correction.

Future work that may be conducted includes the strategic design of pairwise interleavers, the use of trellis termination to achieve increased performance, and the studying of non line-of-sight scenarios for the channel response. The redundant PPM capability in the TTCM system may also be harnessed for an additional mode of error checking.

\section{REFERENCES}

[1] FCC, "New public safety applications and broadband internet access among uses envisioned by fcc authorization of ultra-wideband technology," Unofficial announcement of Commission action, February 2002.

[2] — "Revision of part 15 of the commissions rules regarding ultrawideband transmission systems," Document 00-163, ET Docket No. 98153, April 2002.

[3] Intel, "Ultra-wideband (uwb) technology," September 2007. [Online] Available: http://www.intel.com

[4] M. Z. Win and R. A. Scholtz, "Impulse radio: How it works," in IEEE Communications Letters, vol. 2, no. 2. IEEE, Feb 1998, pp. 36-38.

[5] K. Usuda, H. Zhang, and M. Nakagawa, "Pre-rake performance for pulse based uwb system in a standardized uwb short-range channel," in Wireless Communications and Networking Conference, vol. 2, March 2004, pp. 920-925.

[6] P. Robertson and T. Worz, "Bandwidth-efficient turbo trellis-coded modulation using punctured component codes," in IEEE Journal on Selected Areas in Communications, vol. 16, no. 2, February 1998, pp. 206-218.

[7] S. Lin and D. J. C. Jr, Error Control Coding, 2nd ed. Pearson Prentice Hall, New Jersey, 2004

[8] J. Tan and G. L. Stuber, "A map equivalent sova for non-binary turbo codes," in IEEE International Conference on Communications, vol. 2, June 2000, pp. 602-606.

[9] C. Berrou, M. Jezequel, C. Douillard, and S. Kerouedan, "The advantages of non-binary turbo codes," in ITW2001, Cairns, Australia, September 2001.

[10] K. Popovski, B. J. Wysocki, and T. A. Wysocki, "Modelling and comparative performance analysis of a time reversed uwb system," in to appear in EURASIP Journal on Wireless Communications and Networking, 2007.

[11] A. Swami, B. Sadler, and J. Turner, "On the coexistence of ultrawideband and narrowband radio systems," in Military Communications Conference, Communications for Network-Centric Operations: Creating the Information Force, vol. 1, October 2001, pp. 16-19.

[12] A. Derode, A. Tourin, J. de Rosny, M. Tanter, S. Yon, and M. Fink, "Taking advantage of multiple scattering to communicate with timereversal antennas," Phys. Rev. Lett, vol. 90, no. 1, pp. 014301-1 $014301-4,2003$

[13] A. Saleh and R. Valenzuela, "A statistical model for indoor multipath propagation," IEEE Journal on Selected Areas in Communications, vol. 5, no. 2, pp. 128-137, February 1987.

[14] A. Giulietti, B. Bougard, and L. V. der Perre, Turbo Codes - Desirable and Designable. Kluwer Academic Publishers Group, Massachusetts, USA, 2004.

[15] T. K. Moon, Error Correction Coding - Mathematical Methods and Algorithms. John Wiley \& Sons, New Jersey, 2005.

[16] C. Berrou, R. Pyndiah, P. Adde, C. Douillard, and R. L. Bidan, "An overview of turbo codes and their applications," in The European Conference on Wireless Technology, 3-4 Oct 2005, pp. 1-9.

[17] S. Vafi, "On the design of turbo codes with convolutional interleavers," Ph.D. dissertation, University of Wollongong, 2005.

[18] J. Liu and G. Tu, "Iterative decoding of non-binary turbo codes using symbol based sova algorithm," in International Conference on Соттиnications, Circuits and Systems, vol. 2, June 2006, pp. 689-693.

[19] A. C. Reid, D. P. Taylor, and T. A. Gulliver, "Non-binary turbo codes," in IEEE International Symposium on Information Theory, 2002, p. 57.

[20] L. Cong, W. Xiaofu, and S. Songgeng, "On sova for nonbinary codes," in Fifth Asia-Pacific Conference on Communications and Fourth Optoelectronics and Communications Conference, vol. 1, October 1999, pp. $641-643$.

[21] M.-. G. D. Benedetto and G. Giancola, Understanding Ultra Wide Band - Radio Fundamentals. Prentice Hall, 2004.

[22] Y. Wu, "Matlab code for experiment on turbo codes," MPRG lab, Virginia Tech, 1998. [Online]. Available: http://www.ee.vt.edu/ yufei/turbo.html 